\title{
The Inversion-Free Iterative Methods for Solving the Nonlinear Matrix Equation $X+A^{H} X^{-1} A+B^{H} X^{-1} B=I$
}

\author{
Na Huang and Changfeng Ma \\ School of Mathematics and Computer Science, Fujian Normal University, Fuzhou 350007, China \\ Correspondence should be addressed to Changfeng Ma; macf09@139.com
}

Received 1 August 2013; Accepted 1 November 2013

Academic Editor: Józef Banas

Copyright (C) 2013 N. Huang and C. Ma. This is an open access article distributed under the Creative Commons Attribution License, which permits unrestricted use, distribution, and reproduction in any medium, provided the original work is properly cited.

We present two inversion-free iterative methods for computing the maximal positive definite solution of the equation $X+A^{H} X^{-1} A+$ $B^{H} X^{-1} B=I$. We prove that the sequences generated by the two iterative schemes are monotonically increasing and bounded above. We also present some numerical results to compare our proposed methods with some previously developed inversion-free techniques for solving the same matrix equation.

\section{Introduction}

In this paper, we consider the nonlinear matrix equation:

$$
X+A^{H} X^{-1} A+B^{H} X^{-1} B=I,
$$

where $A, B \in \mathbb{C}^{n \times n}, I$ is the identity matrix, and a Hermitian positive definite solution $X$ is required.

Specifically, if $B=0$, the nonlinear matrix equation (1) reduces to

$$
X+A^{H} X^{-1} A=I .
$$

The nonlinear matrix equation (2) has many applications in nano research, control theory, dynamic programming, statistics, ladder networks, stochastic filtering, and so forth (see [1-7]). The special case (2) has been widely studied by some authors (see [8-27]). Different iterative methods for computing the positive definite solutions of (2) have been proposed, for example, the fixed-point iteration (see [15]), structure-preserving doubling algorithm (see $[7,16])$, and some inversion-free iterations (see [17, 20, 23, 27]). Among them, structure-preserving doubling algorithm has been seen as one of the most efficient algorithms as it has quadratic convergence rate.

However, very little research has been done on the solutions to (1) in the case $B \neq 0$. In [28], Long et al. stated the application background of (1) and presented some conditions for the existence of the positive definite solution of this equation. Moreover, they proposed some iterative algorithms to find the positive definite solution. Popchev et al. made a complete perturbation analysis of (1) (see [29]). In [30], Liu and Chen considered the nonlinear matrix equation $X^{s}+$ $A^{H} X^{-t_{1}} A+B^{H} X^{-t_{2}} B=Q$. They studied the existence of the positive definite solution of this equation.

Motivated and inspired by the works mentioned above, in this paper, we propose two new inversion-free iterative methods for obtaining the maximal positive definite solution of (1). We prove that the sequences generated by the two iterative schemes are monotonically increasing and bounded above. In addition, we also provide some numerical results to illustrate the effectiveness of the proposed algorithm.

Throughout this paper, we use the following notations: for $A \in \mathbb{C}^{n \times n}$, we write $A^{H}, A^{-1}$ and $\|A\|$ to denote the conjugate transpose, the inverse, and the Frobenius norm of the matrix $A$, respectively. For any $A=\left(a_{i j}\right), B=\left(b_{i j}\right)$, we write $A \geq B$ (or $B \leq A)$ if $A-B$ is a Hermitian positive semidefinite matrix. And we write $A>B$ (or $B<A$ ) if $A-B$ is a Hermitian positive definite matrix. We use 0 to denote the zero matrix of size implied by context and $I$ to denote the identity matrix of size implied by context.

This paper is organized as follows. In Section 2, we present two new iterative methods to solve the nonlinear matrix equation (1). In Section 3, the convergence analysis of 
the proposed methods is given. Some numerical experiments are reported in Section 4. Finally, we conclude this paper in Section 5.

\section{New Inversion-Free Iterative Methods}

In this section, we present two new inversion free iterative algorithms for solving problem (1). Let $Y=X^{-1}$; then the nonlinear matrix equation (1) is equivalent to

$$
Y^{-1}+A^{H} Y A+B^{H} Y B=I .
$$

Obviously, if $Y_{*}$ is a Hermitian positive definite solution of (3), we have $0<Y_{*}^{-1} \leq I$. That is, $Y_{*} \geq I$.

Premultiplying and postmultiplying by $Y$ on (3) simultaneously, we get

$$
Y-Y\left(I-A^{H} Y A-B^{H} Y B\right) Y=0 .
$$

Adding $Y$ to both sides of the above equation, we have

$$
Y=2 Y-Y\left(I-A^{H} Y A-B^{H} Y B\right) Y .
$$

Obviously, $Y$ solves (5) if $Y$ is a solution of (3). Conversely, if $Y$ is a nonsingular solution of (5), $Y$ solves (3) as well.

Thus, we just need to solve the matrix equation (5) if we want to get a Hermitian positive definite solution of (3). By this point, we present the following iterative scheme.

Algorithm 1 (an inversion-free iterative algorithm for (1)).

Step 1. Input the matrix $A, B \in \mathbb{C}^{n \times n}$. Take initial matrix $Y_{0}=I$ and tolerance error $\varepsilon \geq 0$. Set $k:=0$.

Step 2. Obtain $Y_{k+1}$ by the following iterative scheme:

$$
Y_{k+1}=2 Y_{k}-Y_{k} Z_{k} Y_{k}
$$

where $Z_{k}=I-A^{H} Y_{k} A-B^{H} Y_{k} B$.

Step 3. Stop if $\left\|Y_{k+1}-Y_{k}\right\|_{F} \leq \varepsilon$. Otherwise, $k:=k+1$, go to Step 2.

Remark 2. If $B=0$, Algorithm 1 reduces to Algorithm 2.1 in [23] with $\alpha=1$ and $Q=I$. Moreover, as $Y_{0}=I$ is a Hermitian matrix, from (6) we know that $Y_{k}$ is also a Hermitian matrix, for all $k \geq 0$.

On the other hand, premultiplying and postmultiplying by $Y$ on (3), respectively, we have

$$
\begin{aligned}
& I+Y A^{H} Y A+Y B^{H} Y B=Y, \\
& I+A^{H} Y A Y+B^{H} Y B Y=Y .
\end{aligned}
$$

Adding the above two equations, we can get

$$
\begin{aligned}
2 I & +Y A^{H} Y A+Y B^{H} Y B \\
& +A^{H} Y A Y+B^{H} Y B Y=2 Y .
\end{aligned}
$$

By some simple calculating, we obtain

$$
\begin{aligned}
Y= & I+\frac{1}{2} \\
& \times\left[Y\left(A^{H} Y A+B^{H} Y B\right)+\left(A^{H} Y A+B^{H} Y B\right) Y\right] .
\end{aligned}
$$

By the above analysis, we know that if $Y$ is a solution of (3), $Y$ is a solution of (9). Now we prove that a Hermitian positive definite solution of (9) is also a solution of (3).

Theorem 3. Let $Y$ be a Hermitian positive definite solution of the nonlinear matrix equation (9). Then $Y$ is also a positive definite solution of the nonlinear matrix equation (3).

Proof. By the nonlinear matrix equation (9), we have

$$
\begin{aligned}
& Y\left(Y^{-1}+A^{H} Y A+B^{H} Y B-I\right) \\
& \quad+\left(Y^{-1}+A^{H} Y A+B^{H} Y B-I\right) Y=0 .
\end{aligned}
$$

This implies that $Y^{-1}+A^{H} Y A+B^{H} Y B-I$ is a solution of the matrix equation $Y H+H Y=0$, where $H \in \mathbb{C}^{n \times n}$ is unknown. Since $Y$ is positive definite, $Y$ and $-Y$ have no common eigenvalue and the matrix equation $Y H+H Y=0$ has a unique solution (see [31]). As 0 solves the equation $Y H+H Y=0$, we must have $Y^{-1}+A^{H} Y A+B^{H} Y B-I=0$. Namely, $Y$ is a positive definite solution of (3).

Thus, we just need to solve the matrix equation (9) if we want to get a Hermitian positive definite solution of (3). By this point, we present the following iterative scheme.

Algorithm 4 (another inversion free iterative algorithm).

Step 1. Input the matrix $A, B \in \mathbb{C}^{n \times n}$. Take initial matrix $Y_{0}=I$ and tolerance error $\varepsilon \geq 0$. Set $k:=0$.

Step 2. Obtain $Y_{k+1}$ by the following iterative scheme:

$$
\begin{gathered}
Z_{k}=\frac{1}{2} Y_{k}\left(A^{H} Y_{k} A+B^{H} Y_{k} B\right), \\
Y_{k+1}=I+Z_{k}^{H}+Z_{k} .
\end{gathered}
$$

Step 3. Stop if $\left\|Y_{k+1}-Y_{k}\right\|_{F} \leq \varepsilon$. Otherwise, $k:=k+1$ go to Step 2.

Obviously, for all $k \geq 0$, sequence $\left\{Y_{k}\right\}$ generated by Algorithm 4 with the initial matrix $Y_{0}=I$ are all Hermitian matrices.

\section{Convergence Analysis}

In this section, we will prove that the sequences $\left\{Y_{k}\right\}$ generated by Algorithms 1 and 4 with the initial matrix $Y_{0}=I$ converge to the minimal positive definite solution of (3). In the first place, we introduce the following lemmas.

Lemma 5 (see [28]). If (1) has a positive definite solution $X$, then $A^{H} A+B^{H} B<I$. 
Lemma 6 (see [23]). If $C$ and $P$ are Hermitian matrices of the same order with $P>0$, then $2 C-C P C \leq P^{-1}$.

Lemma 7. If $M, P \in \mathbb{C}^{n \times n}$ are both Hermitian positive semidefinite, then $M P+P M$ is a Hermitian positive semidefinite matrix as well.

Proof. Obviously, $M P+P M$ is a Hermitian matrix. If one of $M$ and $P$ is positive definite, without loss of generality, suppose that $M>0$. Then by the assumption of the lemma, we know that $M^{1 / 2}, P^{1 / 2}$ are well defined and $M^{1 / 2}>0, P^{1 / 2} \geq 0$. Then we get

$$
\begin{aligned}
M P+P M & =M^{1 / 2} M^{1 / 2} P+P M^{1 / 2} M^{1 / 2} \\
& =M^{1 / 2}\left(M^{1 / 2} P M^{-(1 / 2)}+M^{-(1 / 2)} P M^{1 / 2}\right) M^{1 / 2} \\
& \geq 0 .
\end{aligned}
$$

Assume that neither of $M$ and $P$ is positive definite. Then for $\beta>0$, we have $M+\beta I>0$. Thus by the above analysis, we have $(M+\beta I) P+P(M+\beta I) \geq 0$. Let $\beta \rightarrow 0^{+}$; we get $M P+P M \geq 0$. This completes the proof.

Now we are in a position to prove that $\left\{Y_{k}\right\}$ generated by Algorithm 1 with the initial matrix $Y_{0}=I$ converges to the minimal positive definite solution of (3).

Theorem 8. The nonlinear matrix equation (3) has a positive definite solution and the sequence $\left\{Y_{k}\right\}$ is generated by Algorithm 1 with the initial matrix $Y_{0}=I$. Let $\widetilde{Y}$ be the minimal positive definite solution of (3). Then the sequence $\left\{Y_{k}\right\}$ is well defined, $Y_{0} \leq Y_{1} \leq Y_{2} \leq \cdots<Y_{k} \leq \cdots \leq \tilde{Y}$, and

$$
\lim _{k \rightarrow \infty} Y_{k}=\tilde{Y}
$$

Proof. Let $Y_{*}$ be any positive definite solution of (3). Firstly, we will prove $0<Y_{k} \leq Y_{k+1} \leq Y_{*}$ for all $k \geq 0$ by mathematical induction.

For $k=0$, we have $Y_{0}=I>0$. By (6) we have

$$
\begin{aligned}
Y_{1} & =2 I-I+A^{H} A+B^{H} B \\
& =I+A^{H} A+B^{H} B \geq I=Y_{0} .
\end{aligned}
$$

On the other hand, by Lemma 5 , we have $I-A^{H} A-B^{H} B>0$. This together with Lemma 6 and (3) yields

$$
\begin{aligned}
Y_{1} & =2 I-I\left(I-A^{H} A-B^{H} B\right) I \\
& \leq\left(I-A^{H} A-B^{H} B\right)^{-1} \\
& \leq\left(I-A^{H} Y_{*} A-B^{H} Y_{*} B\right)^{-1} \\
& =Y_{*},
\end{aligned}
$$

where the second inequality follows from the fact that $Y_{*} \geq I$. Hence $0<Y_{k} \leq Y_{k+1} \leq Y_{*}$ holds for $k=0$.
Assume that $0<Y_{k} \leq Y_{k+1} \leq Y_{*}$ holds for $k=i \geq 0$. Since

$$
\begin{aligned}
Z_{i+1} & =I-A^{H} Y_{i+1} A-B^{H} Y_{i+1} B \\
& \geq I-A^{H} Y_{*} A-B^{H} Y_{*} B \\
& =Y_{*}^{-1}>0,
\end{aligned}
$$

by (6), Lemma 6 , and the fact that $Y_{i+1}>0$, we get

$$
Y_{i+2}=2 Y_{i+1}-Y_{i+1} Z_{i+1} Y_{i+1} \leq Z_{i+1}^{-1} \leq Y_{*} .
$$

Moreover, it follows from (6) that

$$
\begin{aligned}
Y_{i+2}-Y_{i+1} & =Y_{i+1}-Y_{i+1} Z_{i+1} Y_{i+1} \\
& =Y_{i+1}\left(Y_{i+1}^{-1}-Z_{i+1}\right) Y_{i+1} .
\end{aligned}
$$

As $Z_{i}=I-A^{H} Y_{i} A-B^{H} Y_{i} B \geq I-A^{H} Y_{i+1} A-B^{H} Y_{i+1} B=$ $Z_{i+1}>0$, this together with (6) and Lemma 6 follows that

$$
Y_{i+1}=2 Y_{i}-Y_{i} Z_{i} Y_{i} \leq Z_{i}^{-1} \leq Z_{i+1}^{-1} .
$$

This implies that $Y_{i+1}^{-1} \geq Z_{i+1}$. Thus, by (18) and the fact that $Y_{i+1}>0$, we obtain $Y_{i+2} \geq Y_{i+1}$. Therefore, $0<Y_{k} \leq Y_{k+1} \leq Y_{*}$ holds for $k=i+1$.

By the principle of mathematical induction, $0<Y_{k} \leq$ $Y_{k+1} \leq Y_{*}$ is true for all $k \geq 0$. The sequence $\left\{Y_{k}\right\}$ is now well defined, monotonically increasing, and bounded above. Let $\lim _{k \rightarrow \infty} Y_{k}=\tilde{Y}$. Then $\widetilde{Y}$ is a positive definite solution of the nonlinear matrix equation (3) by (6). Since $\tilde{Y} \leq Y_{*}$ for any positive definite solution $Y_{*}$ of (3), $\widetilde{Y}$ is the minimal positive definite solution of (3). This completes the proof.

Remark 9. Let $\widetilde{X}$ be the maximal positive definite solution of the nonlinear matrix equation (1). By the relationship between (1) and (3), we get that $\widetilde{X}^{-1}$ is the minimal positive definite solution of (3). So from Theorem 8 , we know that the sequence $\left\{Y_{k}\right\}$ generated by Algorithm 1 with the initial matrix $Y_{0}=I$ converges to the inverse of the maximal positive definite solution of (1).

Now we consider the convergence theorem of Algorithm 4.

Theorem 10. The nonlinear matrix equation (3) has a positive definite solution and the sequence $\left\{Y_{k}\right\}$ is generated by Algorithm 4 with the initial matrix $Y_{0}=I$. Let $\tilde{Y}$ be the minimal positive definite solution of (3). Then the sequence $\left\{Y_{k}\right\}$ is well defined, $Y_{0} \leq Y_{1} \leq Y_{2}<\cdots \leq Y_{k} \leq \cdots \leq \tilde{Y}$, and $\lim _{k \rightarrow \infty} Y_{k}=\tilde{Y}$.

Proof. Let $Y_{*}$ be any positive definite solution of (3). Firstly, we will prove $0<Y_{k} \leq Y_{k+1} \leq Y_{*}$ for all $k \geq 0$ by induction.

For $k=0$, we have $Y_{0}=I>0$; then $Y_{1}=I+A^{H} A+$ $B^{H} B \geq I$. On the other hand, by the fact that $Y_{*} \geq I$, (3), and Lemma 7, we get

$$
\begin{aligned}
Y_{1} & =I+A^{H} A+B^{H} B \\
& \leq I+A^{H} Y_{*} A+B^{H} Y_{*} B
\end{aligned}
$$




$$
\begin{aligned}
= & I+\frac{1}{2}\left(A^{H} Y_{*} A I+I A^{H} Y_{*} A\right) \\
& +\frac{1}{2}\left(B^{H} Y_{*} B I+I B^{H} Y_{*} B\right) \\
\leq & I+\frac{1}{2}\left(A^{H} Y_{*} A Y_{*}+Y_{*} A^{H} Y_{*} A\right) \\
& +\frac{1}{2}\left(B^{H} Y_{*} B Y_{*}+Y_{*} B^{H} Y_{*} B\right) \\
= & I+\frac{1}{2}\left(A^{H} Y_{*} A+B^{H} Y_{*} B\right) Y_{*} \\
& +\frac{1}{2} Y_{*}\left(A^{H} Y_{*} A+B^{H} Y_{*} B\right) \\
= & I+\frac{1}{2}\left(I-Y_{*}^{-1}\right) Y_{*}+\frac{1}{2} Y_{*}\left(I-Y_{*}^{-1}\right) \\
= & Y_{*} \cdot
\end{aligned}
$$

Hence $0<Y_{k} \leq Y_{k+1} \leq Y_{*}$ is true for $k=0$.

Assume that $0<Y_{k} \leq Y_{k+1} \leq Y_{*}$ holds for $k=i \geq 0$. Then by Lemma 7 we have

$$
\begin{gathered}
Y_{i+1} A^{H} Y_{i+1} A+A^{H} Y_{i+1} A Y_{i+1} \\
\geq Y_{i} A^{H} Y_{i+1} A+A^{H} Y_{i+1} A Y_{i} \\
\geq Y_{i} A^{H} Y_{i} A+A^{H} Y_{i} A Y_{i} .
\end{gathered}
$$

Similarly, we get $Y_{i+1} B^{H} Y_{i+1} B+B^{H} Y_{i+1} B Y_{i+1} \geq Y_{i} B^{H} Y_{i} B+$ $B^{H} Y_{i} B Y_{i}$. This together with (11) yields

$$
\begin{aligned}
Y_{i+2}= & I+\frac{1}{2}\left(Y_{i+1} A^{H} Y_{i+1} A+Y_{i+1} B^{H} Y_{i+1} B\right. \\
& \left.+A^{H} Y_{i+1} A Y_{i+1}+B^{H} Y_{i+1} B Y_{i+1}\right) \\
= & I+\frac{1}{2}\left(Y_{i+1} A^{H} Y_{i+1} A+A^{H} Y_{i+1} A Y_{i+1}\right) \\
& +\frac{1}{2}\left(Y_{i+1} B^{H} Y_{i+1} B+B^{H} Y_{i+1} B Y_{i+1}\right) \\
\geq & I+\frac{1}{2}\left(Y_{i} A^{H} Y_{i} A+A^{H} Y_{i} A Y_{i}\right) \\
& +\frac{1}{2}\left(Y_{i} B^{H} Y_{i} B+B^{H} Y_{i} B Y_{i}\right) \\
= & I+\frac{1}{2}\left(Y_{i} A^{H} Y_{i} A+Y_{i} B^{H} Y_{i} B\right. \\
& \left.+A^{H} Y_{i} A Y_{i}+B^{H} Y_{i} B Y_{i}\right) \\
= & I+\frac{1}{2}\left(Z_{i}+Z_{i}^{H}\right)=Y_{i+1} \cdot
\end{aligned}
$$

TABLE 1: Numerical results of Example 11.

\begin{tabular}{lcccc}
\hline Scheme & Iter. & CPU & Res. & MM \\
\hline A1 & 14 & 0.0006 & $3.5756 e-012$ & 84 \\
A2 & 20 & 0.0008 & $2.3117 e-011$ & 100 \\
B1 & 26 & 0.0011 & $4.7298 e-011$ & 156 \\
B2 & 15 & 0.0007 & $6.7304 e-013$ & 90 \\
\hline
\end{tabular}

TABle 2: Numerical results of Example 12.

\begin{tabular}{lcccc}
\hline Scheme & Iter. & CPU & Res. & MM \\
\hline A1 & 48 & 0.0027 & $2.2801 e-011$ & 288 \\
A2 & 83 & 0.0040 & $4.4924 e-011$ & 415 \\
B1 & 92 & 0.0049 & $6.4280 e-011$ & 552 \\
B2 & 49 & 0.0028 & $1.4600 e-011$ & 294 \\
\hline
\end{tabular}

On the other hand, by Lemma 7 we obtain

$$
\begin{aligned}
& Y_{i+1} A^{H} Y_{i+1} A+A^{H} Y_{i+1} A Y_{i+1} \\
& \leq Y_{*} A^{H} Y_{i+1} A+A^{H} Y_{i+1} A Y_{*} \\
& \leq Y_{*} A^{H} Y_{*} A+A^{H} Y_{*} A Y_{*} .
\end{aligned}
$$

Similarly, we get

$$
Y_{i+1} B^{H} Y_{i+1} B+B^{H} Y_{i+1} B Y_{i+1} \leq Y_{*} B^{H} Y_{*} B+B^{H} Y_{*} B Y_{*} .
$$

Using the above inequalities, we can deduce that

$$
\begin{aligned}
Y_{i+2}= & I+\frac{1}{2}\left(Y_{i+1} A^{H} Y_{i+1} A+A^{H} Y_{i+1} A Y_{i+1}\right) \\
& +\frac{1}{2}\left(Y_{i+1} B^{H} Y_{i+1} B+B^{H} Y_{i+1} B Y_{i+1}\right) \\
\leq & I+\frac{1}{2}\left(Y_{*} A^{H} Y_{*} A+A^{H} Y_{*} A Y_{*}\right) \\
& +\frac{1}{2}\left(Y_{*} B^{H} Y_{*} B+B^{H} Y_{*} B Y_{*}\right) \\
= & I+\frac{1}{2} Y_{*}\left(A^{H} Y_{*} A+B^{H} Y_{*} B\right) \\
& +\frac{1}{2}\left(A^{H} Y_{*} A+B^{H} Y_{*} B\right) Y_{*} \\
= & I+\frac{1}{2} Y_{*}\left(I-Y_{*}^{-1}\right)+\frac{1}{2}\left(I-Y_{*}^{-1}\right) Y_{*} \\
= & Y_{*},
\end{aligned}
$$

where the first equality follows from (11) and the third equality follows from (3). Hence, $0<Y_{k} \leq Y_{k+1} \leq Y_{*}$ holds for $k=i+2$.

Thus, by the principle of induction, $0<Y_{k} \leq Y_{k+1} \leq$ $Y_{*}$ is true for all $k \geq 0$. The sequence $\left\{Y_{k}\right\}$ is now well defined, monotonically increasing, and bounded above. Let $\lim _{k \rightarrow \infty} Y_{k}=\tilde{Y}$. Then $\tilde{Y}$ is a positive definite solution of the nonlinear matrix equation (3) by (11) and Theorem 3. Since $\tilde{Y} \leq Y^{H}$ for any positive definite solution $Y^{H}$ of (3), $\widetilde{Y}$ is the minimal positive definite solution of (3). This completes the proof. 
TABLE 3: Numerical results of Example 13.

\begin{tabular}{lcccc}
\hline Scheme & Iter. & CPU & Res. & MM \\
\hline A1 & 28 & 0.0016 & $1.4769 e-011$ & 168 \\
A2 & 47 & 0.0025 & $2.8856 e-011$ & 235 \\
B1 & 54 & 0.0032 & $4.4578 e-011$ & 324 \\
B2 & 29 & 0.0018 & $6.6485 e-012$ & 174 \\
\hline
\end{tabular}

\section{Numerical Experiments}

In this section, we will give some numerical examples to support our Algorithms 1 and 4. All experiments were run on a PC with Pentium(R) Dual-Core CPU E5800 @2.40 GHz. All the programming is implemented in MATLAB R2011b (7.13). We report the number of required iterations (Iter.), the norm of the residual (Res.) when the process is stopped, the required $\mathrm{CPU}$ time (CPU), and the number of matrix-matrix (MM) products required. In our implementation, we stop all considered algorithms when $\left\|Y_{k+1}-Y_{k}\right\|_{F} \leq 10^{-10}$ with $k \geq 1$. We compare our Algorithm 1 (A1) and Algorithm 4 (A2) with the following inverse-free methods for solving (1).

(i) In [28], for finding positive definite solution of (1), Long et al. proposed the following iteration:

$$
\text { B1 : }\left\{\begin{array}{l}
X_{0}=I, \quad Y_{0}=I, \\
X_{k+1}=I-A^{H} Y_{k} A-B^{H} Y_{k} B, \\
Y_{k+1}=Y_{k}\left(2 I-X_{k} Y_{k}\right) .
\end{array}\right.
$$

(ii) In [30], for solving positive definite solution of (1), Liu and Chen proposed the following iteration:

$$
\text { B2 }:\left\{\begin{array}{l}
X_{0}=I, \quad Y_{0}=I, \\
Y_{k+1}=Y_{k}\left(2 I-X_{k} Y_{k}\right), \\
X_{k+1}=I-A^{H} Y_{k+1} A-B^{H} Y_{k+1} B .
\end{array}\right.
$$

Example 11. For the first experiment, we consider (1) when $A$ and $B$ are given as in Example 4.1 from [28]:

$$
\begin{aligned}
A & =\left(\begin{array}{ccc}
0.010 & -0.150 & -0.259 \\
0.015 & 0.212 & -0.064 \\
0.025 & -0.069 & 0.138
\end{array}\right), \\
B & =\left(\begin{array}{ccc}
0.160 & -0.025 & 0.020 \\
-0.025 & -0.288 & -0.060 \\
0.004 & -0.016 & -0.120
\end{array}\right) .
\end{aligned}
$$

We could obtain the maximal solution $X_{+}$(the first 4 digits) by the iterative schemes A1-A2 and B1-B3. The maximal solution is

$$
X_{+} \approx\left(\begin{array}{ccc}
0.9718 & -0.0049 & -0.0046 \\
-0.0049 & 0.8144 & -0.0388 \\
-0.0046 & -0.0388 & 0.8836
\end{array}\right) \text {. }
$$

TABLE 4: Numerical results of Example 14.

\begin{tabular}{lccccc}
\hline DIM & Scheme & Iter. & CPU & Res. & MM \\
\hline \multirow{6}{*}{64} & A1 & 16 & 0.0050 & $1.4358 e-011$ & 96 \\
& A2 & 24 & 0.0070 & $1.4344 e-011$ & 120 \\
& B1 & 32 & 0.0419 & $3.1053 e-011$ & 192 \\
& B2 & 17 & 0.0064 & $3.6219 e-012$ & 102 \\
\hline \multirow{6}{*}{128} & A1 & 17 & 0.0324 & $4.9074 e-012$ & 102 \\
& A2 & 24 & 0.0620 & $1.9656 e-011$ & 120 \\
& B1 & 32 & 0.1036 & $4.0817 e-011$ & 192 \\
& B2 & 18 & 0.0454 & $1.2594 e-012$ & 108 \\
\hline \multirow{6}{*}{256} & A1 & 17 & 0.3721 & $4.4572 e-012$ & 102 \\
& A2 & 24 & 0.4954 & $1.7529 e-011$ & 120 \\
& B1 & 32 & 0.6902 & $3.7397 e-011$ & 192 \\
512 & B2 & 18 & 0.4155 & $1.1373 e-012$ & 108 \\
\hline \multirow{6}{*}{5} & A1 & 17 & 2.5991 & $5.1113 e-012$ & 168 \\
& A2 & 24 & 2.9816 & $2.0378 e-011$ & 235 \\
& B1 & 32 & 4.4817 & $4.2332 e-011$ & 324 \\
& B2 & 18 & 2.7676 & $1.3148 e-012$ & 174 \\
\hline \multirow{6}{*}{1024} & A1 & 17 & 20.2298 & $4.9186 e-012$ & 168 \\
& A2 & 24 & 26.0464 & $1.9494 e-011$ & 235 \\
& B1 & 32 & 36.6167 & $4.0910 e-011$ & 324 \\
& B2 & 18 & 21.8226 & $1.2626 e-012$ & 174 \\
\hline
\end{tabular}

Example 12. In this test, the matrices $A$ and $B$ are given as in Example 4.2 from [28]:

$$
\begin{aligned}
A & =\frac{1}{680}\left(\begin{array}{lllll}
40 & 25 & 23 & 35 & 66 \\
25 & 32 & 27 & 45 & 21 \\
23 & 27 & 28 & 16 & 24 \\
35 & 45 & 16 & 52 & 65 \\
66 & 21 & 24 & 65 & 69
\end{array}\right), \\
B & =\frac{1}{400}\left(\begin{array}{lllll}
11 & 21 & 23 & 25 & 32 \\
21 & 31 & 60 & 42 & 33 \\
23 & 60 & 34 & 18 & 26 \\
25 & 42 & 18 & 44 & 30 \\
32 & 33 & 26 & 30 & 50
\end{array}\right) .
\end{aligned}
$$

We will obtain the maximal solution $X_{+}$(the first 4 digits) by the iterative schemes $\mathrm{A} 1-\mathrm{A} 2$ and $\mathrm{B} 1-\mathrm{B} 3$. The maximal solution is

$$
X_{+}=\left(\begin{array}{ccccc}
0.9437 & -0.0642 & -0.0530 & -0.0691 & -0.0772 \\
-0.0642 & 0.9063 & -0.0739 & -0.0833 & -0.0907 \\
-0.0530 & -0.0739 & 0.9297 & -0.0717 & -0.0763 \\
-0.0691 & -0.0833 & -0.0717 & 0.9080 & -0.0970 \\
-0.0772 & -0.0907 & -0.0763 & -0.0970 & 0.8889
\end{array}\right)
$$

Our numerical results are reported in Table 1.

Our numerical results are reported in Table 2. 
Example 13. In this experiment we solve (1) with the matrices $A$ and $B$ as follows:

$$
A=\frac{1}{20}\left(\begin{array}{llllll}
2 & 0 & 0 & 1 & 0 & 0 \\
1 & 2 & 0 & 0 & 1 & 0 \\
0 & 0 & 3 & 0 & 1 & 0 \\
1 & 0 & 0 & 2 & 0 & 1 \\
1 & 0 & 1 & 0 & 3 & 0 \\
0 & 1 & 0 & 0 & 1 & 2
\end{array}\right)
$$

$$
B=\frac{1}{50}\left(\begin{array}{llllll}
2 & 1 & 6 & 0 & 5 & 7 \\
3 & 4 & 7 & 1 & 3 & 0 \\
0 & 9 & 2 & 4 & 7 & 8 \\
8 & 5 & 3 & 0 & 0 & 1 \\
2 & 5 & 0 & 2 & 1 & 7 \\
4 & 0 & 0 & 1 & 4 & 9
\end{array}\right)
$$

We could obtain the maximal solution $X_{+}$(the first 4 digits) by the iterative schemes $\mathrm{A} 1-\mathrm{A} 2$ and $\mathrm{B} 1-\mathrm{B} 3$. The unique positive definite solution is

$$
X_{+} \approx\left(\begin{array}{cccccc}
0.9301 & -0.0443 & -0.0367 & -0.0202 & -0.0411 & -0.0521 \\
-0.0443 & 0.9104 & -0.0395 & -0.0271 & -0.0603 & -0.0781 \\
-0.0367 & -0.0395 & 0.9234 & -0.0110 & -0.0566 & -0.0430 \\
-0.0202 & -0.0271 & -0.0110 & 0.9755 & -0.0224 & -0.0374 \\
-0.0411 & -0.0603 & -0.0566 & -0.0224 & 0.9064 & -0.0858 \\
-0.0521 & -0.0781 & -0.0430 & -0.0374 & -0.0858 & 0.8486
\end{array}\right)
$$

The numerical results are listed in Table 3.

Example 14. In this test, the matrices $A$ and $B$ are provided with the following forms:

$$
A=\frac{1}{10} I_{n}+\frac{1}{2 n} R_{n}, \quad B=\frac{1}{20} I_{n}+\frac{1}{n^{2}} S_{n}
$$

where $I_{n}$ is $n \times n$ identity matrix and $R_{n}=\left(r_{i j}\right)_{n \times n}$ and $S_{n}=$ $\left(s_{k l}\right)_{n \times n}$ are randomly generated with entries $r_{i j} \in(0,1)$ and $s_{k l} \in(-1,1)$. For different matrix dimension (DIM) $n$, the numerical results are reported in Table 4 .

From the above experiments, we find that Algorithm 1 has an advantage in the number of iterations and CPU time. Although Algorithm 4 performs worse than B2, it performs better than B1. In Example 14, we can see that the iterations of Algorithms 1 and 4 are invariant with the dimension, which are the same as the performance of B1 and B2. In a word, our algorithms are promising.

\section{Conclusion}

In this paper, we propose two inversion-free iterative algorithms for obtaining the maximal positive definite solution of the equation $X+A^{H} X^{-1} A+B^{H} X^{-1} B=I$. We prove that the sequences generated by the proposed iterative schemes are monotonically increasing and bounded above. Some numerical results are also reported in the paper, which confirm the good theoretical properties of our approach. Although we prove that the sequence $\left\{Y_{k}\right\}$ generated by Algorithms 1 and 4 with the initial matrix $Y_{0}=I$ converges to the minimal positive definite solution of (3) (or the maximal positive definite solution of (1)), we do not yet give the analysis on the convergence rate of the two methods, which is our work in the future.

\section{Conflict of Interests}

The authors declare that there is no conflict of interests regarding the publication of this paper.

\section{Authors' Contribution}

All authors contributed equally and significantly in writing this paper. All authors read and approved the final paper.

\section{Acknowledgments}

The project is supported by the National Natural Science Foundation of China (Grant nos. 11071041 and 11201074), Fujian Natural Science Foundation (Grant No. 2013J01006) and The University Special Fund Project of Fujian (Grant no. JK2013060).

\section{References}

[1] J. C. Engwerda, "On the existence of a positive definite solution of the matrix equation $X+A^{T} X^{-1} A=I$," Linear Algebra and Its Applications, vol. 194, pp. 91-108, 1993.

[2] X. Zhan and J. Xie, "On the matrix equation $X+A^{T} X^{-1} A=I$," Linear Algebra and Its Applications, vol. 247, pp. 337-345, 1996.

[3] W. N. Anderson, Jr., T. D. Morley, and G. E. Trapp, "Positive solutions to $X=A-B X^{-1} B^{x}$," Linear Algebra and Its Applications, vol. 134, pp. 53-62, 1990.

[4] A. Ferrante and B. C. Levy, "Hermitian solutions of the equation $X=Q+N X^{-1} N^{x}$," Linear Algebra and Its Applications, vol. 247, pp. 359-373, 1996.

[5] P. Lancaster and L. Rodman, Algebraic Riccati Equations, Oxford University Press, Oxford, UK, 1995.

[6] C.-H. Guo, Y.-C. Kuo, and W.-W. Lin, "On a nonlinear matrix equation arising in nano research," SIAM Journal on Matrix Analysis and Applications, vol. 33, no. 1, pp. 235-262, 2012.

[7] C.-H. Guo and W.-W. Lin, "The matrix equation $X+A^{T} X^{-1} A=$ $Q$ and its application in nano research," SIAM Journal on Scientific Computing, vol. 32, no. 5, pp. 3020-3038, 2010. 
[8] V. I. Hasanov and I. G. Ivanov, "Solutions and perturbation estimates for the matrix equations $X \pm A^{x} X^{-n} A=Q$," Applied Mathematics and Computation, vol. 156, no. 2, pp. 513-525, 2004.

[9] C.-H. Guo and P. Lancaster, "Iterative solution of two matrix equations," Mathematics of Computation, vol. 68, no. 228, pp. 1589-1603, 1999.

[10] S. M. El-Sayed and A. C. M. Ran, "On an iteration method for solving a class of nonlinear matrix equations," SIAM Journal on Matrix Analysis and Applications, vol. 23, no. 3, pp. 632-645, 2001.

[11] S. M. El-Sayed, "Two sided iteration methods for computing positive definite solutions of a nonlinear matrix equation," Journal of the Australian Mathematical Society B, vol. 44, pp. 1-8, 2003.

[12] S. M. El-Sayed and A. M. Al-Dbiban, "On positive definite solutions of the nonlinear matrix equation," Applied Mathematics and Computation, vol. 151, no. 2, pp. 533-541, 2004.

[13] X. Zhan, "Computing the extremal positive definite solutions of a matrix equation," SIAM Journal on Scientific Computing, vol. 17, no. 5, pp. 1167-1174, 1996.

[14] C.-H. Guo, Y.-C. Kuo, and W.-W. Lin, "Complex symmetric stabilizing solution of the matrix equation $X+A^{T} X^{-1} A=Q$," Linear Algebra and Its Applications, vol. 435, no. 6, pp. 1187-1192, 2011.

[15] J. C. Engwerda, A. C. M. Ran, and A. L. Rijkeboer, "Necessary and sufficient conditions for the existence of a positive definite solution of the matrix equation $X+A^{H} X^{-1} A=Q$," Linear Algebra and Its Applications, vol. 186, pp. 255-275, 1993.

[16] W.-W. Lin and S.-F. Xu, "Convergence analysis of structurepreserving doubling algorithms for Riccati-type matrix equations," SIAM Journal on Matrix Analysis and Applications, vol. 28, no. 1, pp. 26-39, 2006.

[17] S. M. El-Sayed and A. M. Al-Dbiban, "A new inversion free iteration for solving the equation $X+A^{H} X^{-1} A=Q$," Journal of Computational and Applied Mathematics, vol. 181, no. 1, pp. 148-156, 2005.

[18] B. Meini, "Efficient computation of the extreme solutions of $X+A^{H} X^{-1} A=Q$ and $X-A^{H} X^{-1} A=Q$," Mathematics of Computation, vol. 71, no. 239, pp. 1189-1204, 2002.

[19] X. Yin and S. Liu, "Positive definite solutions of the matrix equations $X \pm A^{H} X^{-q} A=Q(q \geq 1)$," Computers \& Mathematics with Applications, vol. 59, no. 12, pp. 3727-3739, 2010.

[20] M. Monsalve and M. Raydan, "A new inversion-free method for a rational matrix equation," Linear Algebra and Its Applications, vol. 433, no. 1, pp. 64-71, 2010.

[21] J. Tomfohr and O. F. Sankey, "Theoretical analysis of electron transport through organic molecules," Journal of Chemical Physics, vol. 120, pp. 1542-1554, 2004.

[22] I. G. Ivanov and S. M. El-sayed, "Properties of positive definite solutions of the equation $X+A^{H} X^{-2} A=I$," Linear Algebra and Its Applications, vol. 279, no. 1-3, pp. 303-316, 1998.

[23] Z.-y. Peng, S. M. El-Sayed, and X.-l. Zhang, "Iterative methods for the extremal positive definite solution of the matrix equation $X+A^{H} X^{-\alpha} A=Q$," Journal of Computational and Applied Mathematics, vol. 200, no. 2, pp. 520-527, 2007.

[24] S. M. El-Sayed, "Two iteration processes for computing positive definite solutions of the equation $X-A^{H} X^{-n} A=Q$," Computers \& Mathematics with Applications, vol. 41, no. 5-6, pp. 579-588, 2001.
[25] A. M. Al-Dubiban, "On the iterative method for the system of nonlinear matrix equations," Abstract and Applied Analysis, vol. 2013, Article ID 685753, 7 pages, 2013.

[26] A. M. Al-Dubiban, "Iterative algorithm for solving a system of nonlinear matrix equations," Journal of Applied Mathematics, vol. 2012, Article ID 461407, 15 pages, 2012.

[27] L. Zhang, "An improved inversion-free method for solving the matrix equation $X+A^{x} X^{-\alpha} A=Q$," Journal of Computational and Applied Mathematics, vol. 253, pp. 200-203, 2013.

[28] J.-h. Long, X.-y. Hu, and L. Zhang, "On the Hermitian positive definite solution of the nonlinear matrix equation $X+A^{H} X^{-1} A+$ $B^{H} X^{-1} B=I$," Bulletin of the Brazilian Mathematical Society, vol. 39, no. 3, pp. 371-386, 2008.

[29] I. Popchev, P. Petkov, M. Konstantinov, and V. Angelova, "Perturbation bounds for the bonlinear matrix equation," in Large-Scale Scientific Computing, vol. 7116 of Lecture Notes in Computer Science, pp. 155-162, 2012.

[30] A. J. Liu and G. L. Chen, "On the Hermitian positive defnite solutions of nonlinear matrix equation $X^{s}+A^{*} X^{-t_{2}} A+$ $B^{*} X^{-t_{2}} B=$ Q," Mathematical Problems in Engineering, vol. 2011, Article ID 163585, 18 pages, 2011.

[31] P. C. Müller, "Solution of the matrix equations $A X+X B=-Q$ and $S^{T} X+X S=-Q$," SIAM Journal on Applied Mathematics, vol. 18 , pp. 682-687, 1970. 


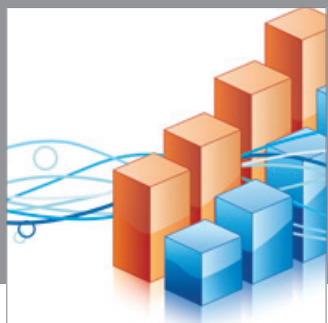

Advances in

Operations Research

mansans

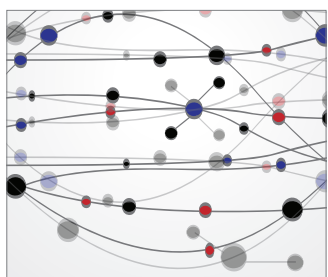

The Scientific World Journal
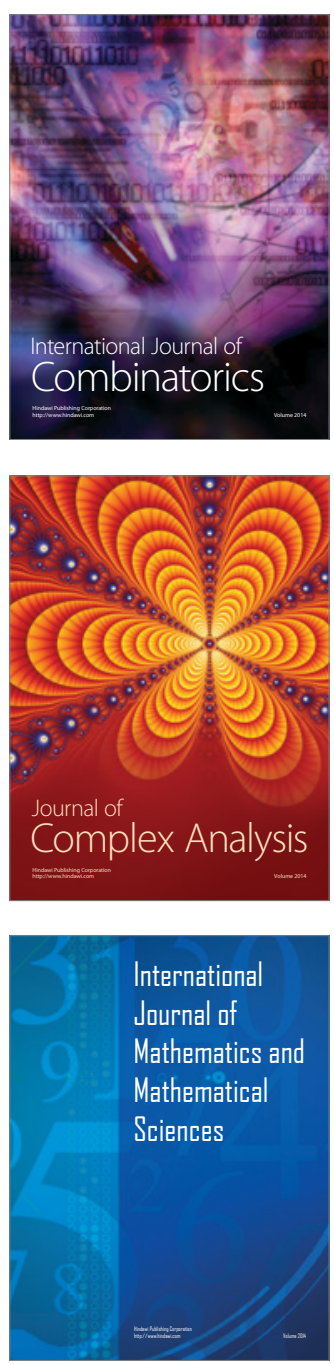
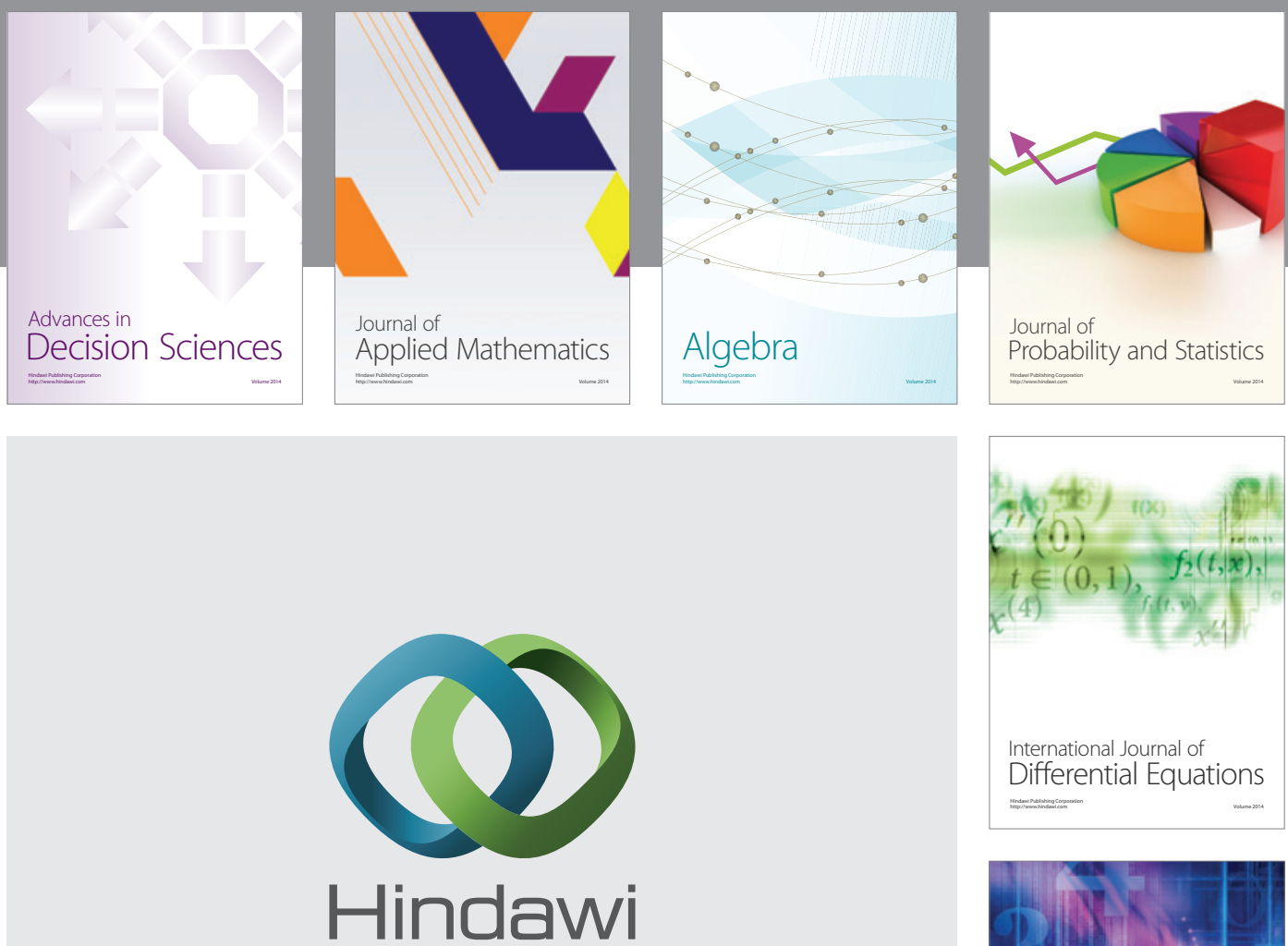

Submit your manuscripts at http://www.hindawi.com
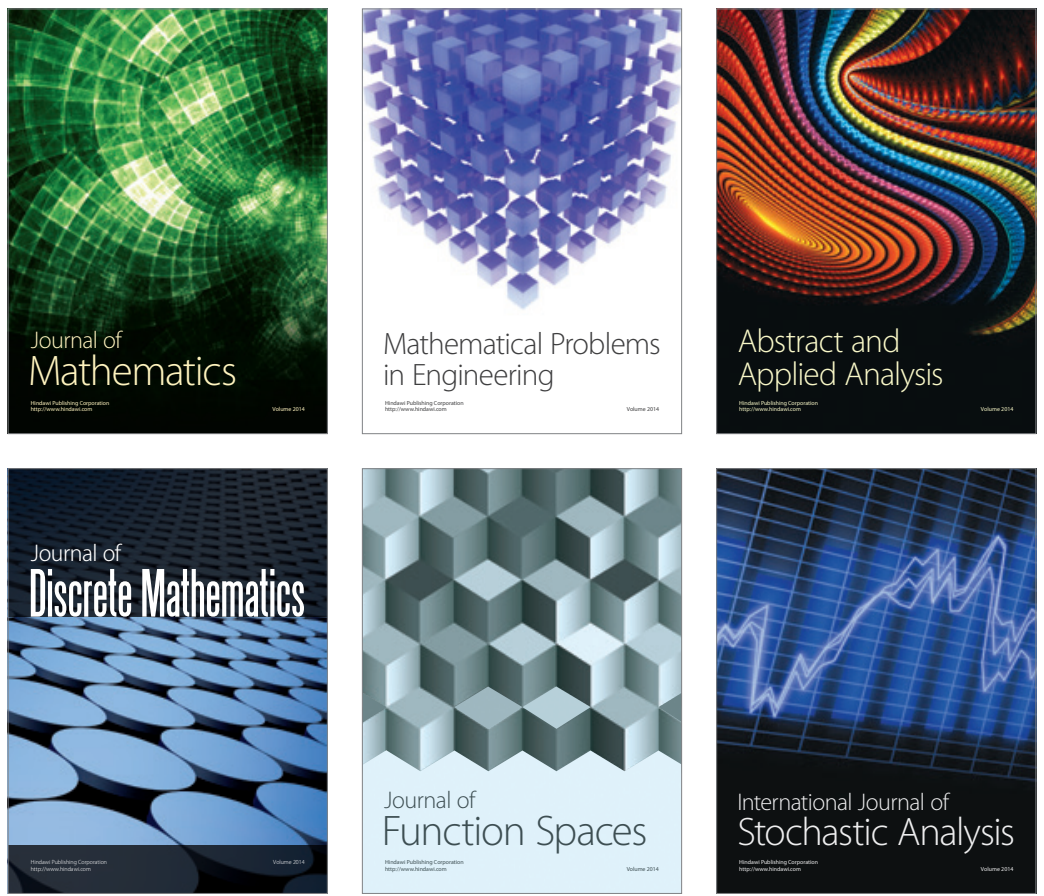

Journal of

Function Spaces

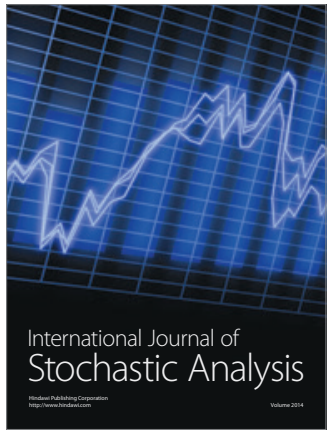

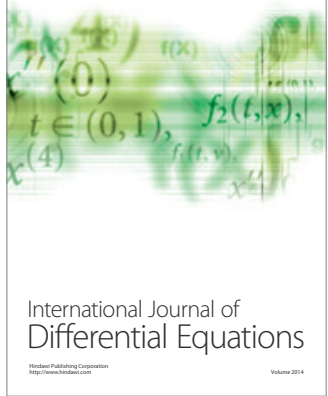
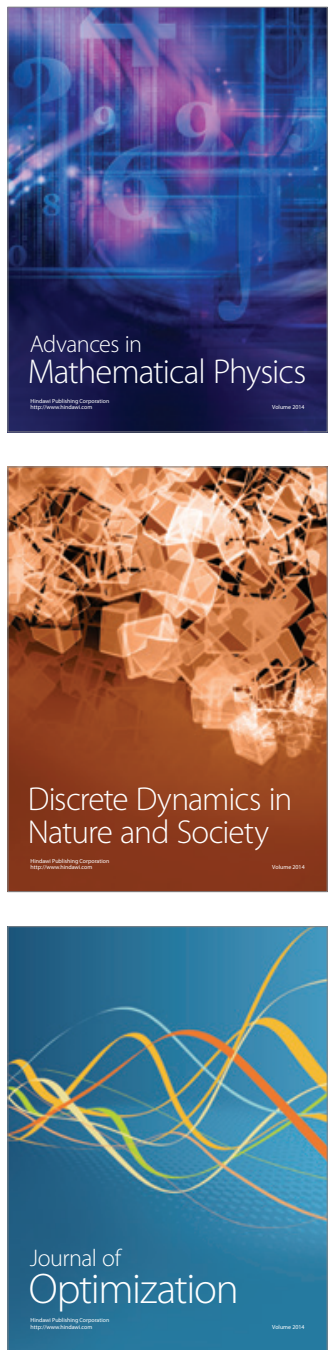